\title{
Tendances de la géographie scolaire en Suisse romande Quelques réflexions d'ordre didactique
}

\section{Introduction}

Lobjet de cet article est de présenter les tendances que l'on peut déceler dans la géographie scolaire romande d'aujourd'hui. A cet effet, je passerai rapidement en revue les programmes des cantons romands, en me limitant au cadre secondaire pour ce qui est des dernières années de la scolarité obligatoire. Cet inventaire n'aurait qu'une utilité médiocre s'il ne pouvait servir de base à une réflexion d'ordre didactique. Celle-ci m'amènera à exposer quelquesunes des pistes sur lesquelles il paraît souhaitable d'engager la géographie scolaire pour dynamiser cette discipline.

Une bonne partie de ces réflexions repose sur des publications parues récemment en France et en Belgique. Des travaux remarquables paraissent depuis près quinze ans en Belgique, grâce à quelques chercheurs et praticiens de la didactique de la géographie. Certains de ces travaux ont d'ailleurs en partie orienté les programmes romands. En France par contre, si l'on excepte le livre de DEBESSE-ARVISET paru en 1969 et l'originalité de la démarche des manuels auxquels a collaboré Y. Lacoste, une véritable réflexion sur la didactique de la géographie ne s'est engagée que depuis le début des années quatrevingts et semble avoir été induite par la profonde crise que connaît dans ce pays l'enseignement de la géographie. Il faudra attendre 1986 pour que trois revues consacrent une large place à des travaux relevant du domaine didactique.

Le présent article permettra ainsi d'évoquer certaines tendances de la didactique de la géographie dans l'aire francophone, qui influencent dans une certaine mesure la pratique de l'enseignement géographique dans les cantons romands (1).

(1) La première partie et quelques autres éléments de cet article sont un résumé très succinct d'une conférence tenue le 30 août 1988 par l'auteur à Coire, dans le cadre du cours CPS «Rahmenlehrplan Geographie» (29 au 31 août 1988).

\section{Tendances de la géographie scolaire dans les cantons romands}

Il est possible d'appréhender les tendances actuelles de la géographie scolaire romande par le biais de la lecture des programmes d'enseignement de la discipline. Il n'est évidemment pas question d'énumérer ici les détails de chaque programme cantonal, ce qui serait long et ennuyeux, mais plutôt d'effectuer un survol synthétique de leur contenu.

Tous les cantons romands appliquent le même programme pour les quatre premières années de la scolarité obligatoire. De la première à la troisième année, la géographie est englobée, avec les sciences naturelles et l'histoire, dans un ensemble appelé «connaissance de l'environnement». Le support spatial de cette étude part du monde familier de l'enfant en première année, pour s'étendre au village ou au quartier en deuxième et aux environs du village ou à la ville entière en troisième. En quatrième année, les trois branches sont dissociées et font dès lors l'objet d'un enseignement séparé. La région est le cadre dans lequel sont conduites les observations en géographie. Les programmes de ces quatre années visent tous à permettre à l'enfant de prendre conscience de son environnement et d'acquérir les tout premiers savoir-faire nécessaires à l'étude de cet environnement.

Dès la cinquième année, l'unité est moins nette: là commence pour certains cantons l'école secondaire, alors qu'ailleurs elle ne débute qu'en sixième ou septième. Pour les cinquième et sixième années, on peut cependant relever que le support spatial commun à tous les programmes romands est la Suisse et qu'une tendance générale est bien marquée, celle

Philippe Hertig, maître à l'Etablissement Secondaire du Belvédère et collaborateur à la didactique de la géographie au Séminaire Pédagogique de l'Enseignement Secondaire; Chemin du Boisy 49A, 1004 Lausanne. 
de l'étude des paysages. Le paysage est à comprendre ici comme l'aspect visible, directement perceptible, des types d'organisation de l'espace, lesquels sont, avec leur répartition à la surface de la Terre, l'objet d'étude de la géographie. La majorité des cantons répartissent cet enseignement de manière homogène sur les deux années, et proposent de travailler sur des paysages des trois régions naturelles, et des paysages agricoles, industriels et urbains. Berne (2) y ajoute l'étude spécifique de la carte. Seuls Neuchâtel et Vaud se distinguent, en proposant une année d'étude de paysages (cinquième) et une année de géographie thématique de la Suisse (sixième), la formulation de leurs programmes respectifs différant quelque peu sur les objectifs et les contenus.

De la septième à la neuvième année, l'unité est apparemment moins nette encore. Une tendance reste cependant commune à tous les programmes, celle de l'élargissement progressif du support spatial de l'étude. Ainsi travaille-t-on l'Europe en septième année (exceptions: Fribourg, où l'Europe est étudiée en huitième, et Neuchâtel, sur le cas duquel je reviendrai) et les autres continents dans un ordre variable en huitième et neuvième (exceptions: Neuchâtel encore, et Fribourg qui place l'Afrique en septième). Certains programmes imposent l'étude de la Terre: forme, dimensions, géophysique, cosmographie (Berne, Jura). Si l'on passe sur des formulations différentes d'un canton à l'autre et sur l'ordonnance des supports spatiaux, on constate qu'une autre tendance se dessine: la géographie pratiquée est une géographie essentiellement thématique et basée sur des supports spatiaux régionaux, avec des définitions assez fluctuantes pour cette dernière notion. Mais cette tendance commune recouvre en fait des pratiques originales, que les trois exemples ci-après permettront d'illustrer.

Genève met ainsi l'accent, en septième année, sur la notion de «milieu»: dans le cadre spatial de l'Europe, on étudie l'homme et le milieu naturel, le milieu rural et le milieu urbain. En huitième, il est question de la notion d'«espace»: on s'occupe de l'homme et des espaces d'habitation, de production et de consommation, dans les pays industrialisés en général. En neuvième enfin, c'est la notion de «conditions» qui est mise en exergue: le Tiers Monde est le support spatial d'une étude des conditions de vie, des conditions de l'agriculture, de l'industrie et du développement. Les instructions officielles comportent une liste très détaillée d'objectifs généraux et spécifiques pour chaque thème et es-

(2) Chaque fois qu'ils sera question de Berne ou de Fribourg dans le cadre de cet article, il s'agira bien sûr de la partie francophone de ces cantons. pace étudié. De même, des objectifs généraux et spécifiques sont définis quant aux outils géographiques que l'élève doit maîtriser.

Dans le canton de Neuchâtel, un «cycle» d'enseignement de la géographie couvre les septième et huitième années. Dans le but de parvenir à une connaissance et à une compréhension aussi complètes que possible de la répartition des phénomènes géographiques à la surface de la Terre, le programme prévoit l'étude de sept grands thèmes, chacun étant inscrit dans une région du monde (population/Amérique latine, relief/Japon, hydrographie/ Asie, habitat/Afrique, climat/Asie des moussons, économie/Europe et Tiers Monde, communications/URSS et Amérique du Nord). Les régions indiquées doivent être considérées comme des points de départ, à partir desquels il sera procédé à de fréquentes comparaisons avec d'autres parties du monde et avec la Suisse. On perçoit donc une volonté expresse d'inscrire la géographie dans une perspective globale. En neuvième par contre, la géographie est incorporée, avec l'histoire et le civisme, à un «Séminaire d'éducation civique».

Dans le canton de Vaud enfin, il est mis l'accent sur la notion de «paysage». De la septième à la neuvième année, on étudie des «espaces caractéristiques par l'analyse de paysages, permettant la mise en relation de phénomènes de géographie physique, climatique, rurale, urbaine et économique». Ce travail doit à chaque fois déboucher sur une vue d'ensemble mettant en évidence les relations entre «les grands traits du cadre physique, humain et économique» (citations extraites du programme officiel). En outre, en huitième, avec l'URSS, les USA et l'Amérique latine en tant que support spatial, il s'agit de comparer les systèmes économiques capitaliste et socialiste et leur interdépendance avec les pays en voie de développement. En neuvième enfin, chaque étude de paysage doit se prolonger par l'analyse d'un thème de géographie générale, propre au paysage en question et étudié au plan mondial (exemple: le Sahel et la désertification, puis la désertification dans le monde). Ce thème devrait, dans un troisième temps, être replacé dans le contexte suisse, et les vues d'ensemble se faire à l'échelle des continents (Afrique, Asie, Océanie).

Au niveau du secondaire supérieur (gymnases), on note une tendance générale: tous les programmes cantonaux dont j'ai pu avoir connaissance sont des programmes de géographie générale thématique visant à satisfaire, dans la mesure du nombre de périodes hebdomadaires disponibles (très variable d'un canton à l'autre, et parfois même à l'intérieur d'un canton!), les exigences de l'actuel programme de Maturité fédérale. Une grande liberté d'appréciation et de choix est laissée aux maîtres, ce qui permet à certains d'entre eux de concevoir des ensei- 
gnements qui se situent dans une perspective proche de celle suivie au secondaire inférieur (c'est notamment le cas pour Neuchâtel et Vaud, d'après des communications orales de MM. P.-Y. Theurillat et J.-P. Milliet). Seul Fribourg, à ma connaissance, prévoit une année pendant laquelle la géographie régionale figure expressément au programme: on travaille la Suisse, les USA et l'URSS, ces espaces n'étant pas seulement étudiés isolément, mais par exemple dans leurs interrelations, ou dans leurs relations avec les pays en voie de développement.

Ce rapide survol des programmes d'enseignement de la géographie dans les cantons romands permet de recenser quatre tendances principales. Au début de la scolarité obligatoire, la discipline est intégrée à ce que l'on peut appeler l'éducation à la perception ou les activités d'éveil. Dans une perspective assez proche, le travail est ensuite orienté, pour une ou deux années, sur l'analyse des paysages. Puis on passe à une géographie thématique, inscrite en général dans un cadre régional. Sur ces trois grandes tendances se greffe la quatrième, qui est le principe de l'élargissement progressif du support spatial de l'étude. Enfin, hors du cadre de la scolarité obligatoire, la géographie thématique domine largement les programmes des gymnases, alors que les Ecoles normales préparent les instituteurs à un enseignement de «connaissance de l'environnement». Audelà de ces points communs, la formulation exacte des programmes varie d'un canton à l'autre, tant pour les objectifs que pour les contenus. C'est bien évidemment le cas aussi de l'enseignement luimême, ce d'autant plus que les manuels utilisés diffèrent également.

\section{Quelques réflexions d'ordre didactique}

Quel est dès lors le regard que l'on peut porter, d'un point de vue didactique, sur ces différents programmes? Avant de répondre, il convient de définir ce que l'on entend par «didactique». Parmi d'autres, la définition de la didactique de la géographie que donne DAUDEL me semble intéressante: "La didactique de la géographie (...) est la science de l'étude des modalités d'apprentissage du savoir disciplinaire en question. Elle associe, étroitement, le champ de la pratique et celui de la théorie, selon un mode de type recherche-action» (DAUDEL 1986). Je me permets de renvoyer le lecteur qui en souhaiterait une explicitation détaillée, notamment pour la notion de «recherche-action», à l'article riche et dense cité en référence, et particulièrement à l'organigramme illustrant cette définition (p. 142).

Les programmes évoqués ci-dessus répondent-ils donc à la double exigence d'être des cadres permettant un bon apprentissage du savoir géographique et, simultanément, des cadres dans lesquels s'effec- tue la transposition du savoir savant en objet d'enseignement?

Sans entrer dans une critique détaillée de ces programmes, il est possible d'y relever matière à quelques problèmes. On peut, par exemple, discuter la pertinence de l'année consacrée à l'étude thématique de la géographie de la Suisse dans le programme vaudois, qui pour le reste est construit selon une démarche cohérente centrée sur la notion de paysage. On peut se demander pourquoi la géographie en tant que telle est absente de la neuvième année neuchâteloise, alors qu'elle reparaît au gymnase. L'articulation du programme fribourgeois est discutable si l'on admet l'idée de l'élargissement progressif du support spatial. La distinction entre «milieu de vie» et «conditions de vie», dans les septième et neuvième années genevoises, n'est pas très claire. De l'avis de quelques maîtres valaisans, les programmes du secondaire de leur canton rompent avec la logique des programmes primaires et reposent sur des conceptions archaïques. Quant aux notions de cosmographie présentes dans les programmes bernois et jurassien, relèvent-elles vraiment du domaine de la géographie? La géographie thématique pratiquée au gymnase ne risque-t-elle pas de faire double emploi avec ce qui est étudié pendant les dernières années de la scolarité obligatoire? D'une manière plus générale, le concept de «paysage» luimême, pourtant largement utilisé, ne se distingue pas toujours clairement de celui d'espace. Plus fondamentalement encore, on peut se demander si le fractionnement de la géographie en géographie physique, climatique, urbaine, etc., a un sens, et il serait sans doute fructueux de discuter toutes les implications du choix, en soi justifiable, de la géographie thématique inscrite dans un cadre régional, tendance généralisée dès la septième année: cette géographie-là est-elle la suite, la résultante logique de la géographie-branche d'éveil des premières années? Et si l'on inventorie les manuels associés à tel ou tel programme, on peut aussi émettre quelques doutes quant au bien-fondé de certains choix: personnellement, je ne pense par exemple pas que la «Géographie des continents» d'Oskar Bär soit le manuel convenant aux programmes des huitième et neuvième années vaudoises, la qualité intrinsèque de l'ouvrage n'étant d'ailleurs pas mise en cause ici.

Dans un article rédigé à l'occasion du cours du CPS «Aspects de la didactique de la géographie», qui s'est déroulé à Lausanne du 30 mai au 1er juin 1988, Mme MERENNE-SCHOUMAKER relève que les programmes de géographie scolaire de nombreux pays manquent de cohésion interne, et qu'on peut y constater une absence de gradation dans les apprentissages et les objectifs: «Tout se passe comme si on avait juxtaposé différentes matières sans s'interroger sur les prérequis à certaines acquisitions et sur les difficultés inhérentes à certains contenus. A la limite, on 
est tenté de croire qu'il serait possible d'intervertir les programmes de certaines années sans ébranler profondément la formation» (MERENNE-SCHOUMAKER 1988, inédit). Le jugement est sévère; mais il a le mérite de mettre en évidence l'absolue nécessité d'une meilleure cohérence des programmes scolaires. Et, d'une manière ou d'une autre, les programmes romands sont sans doute tous perfectibles.

Il n'est d'ailleurs pas question ici d'affirmer que ces programmes sont tout simplement incohérents. On est heureusement loin, désormais, de ces affligeantes énumérations de noms de villes, montagnes, lacs, rivières ou cols qui, avec les cartes muettes, constituaient il n'y pas si longtemps encore l'essentiel de la géographie scolaire. On a également, du moins en principe, dépassé l'étude de portions d'espace selon leurs frontières politiques. On peut aussi estimer que les programmes romands sont beaucoup plus cohérents que ceux proposés en France, qui souffrent considérablement de l'intégration de l'histoire et de la géographie dans un ensemble dit des «sciences sociales»: on va en France jusqu'à parler d'un ensemble «histoire-géographie-économie-instruction civique», et la logique veut que les élèves ne disposent que d'un seul manuel couvrant toutes ces disciplines jusqu'à la fin du premier cycle secondaire (secondaire inférieur); ainsi, on «calque» la structure du programme de géographie, et plus précisément les supports spatiaux de l'étude, sur les espaces déjà évoqués au travers du programme d'histoire. Si la géographie proposée aux écoliers romands du secondaire inférieur n'est heureusement pas aussi insatisfaisante, il n'en reste pas moins que l'on peut s'interroger à juste titre sur certaines orientations de nos programmes.

Ces interrogations mènent immanquablement au problème de la définition de la géographie. Comment, en effet, concevoir un enseignement sans une réflexion sur ce qui doit être enseigné? Chaque maître est constamment confronté à la question de savoir comment «faire passer» telle connaissance, comment en évaluer l'acquisition. Ce sont là des problèmes d'ordre pédagogique. Mais il peut se demander, et ce même dans le cadre d'un programme qui lui est imposé, quelles connaissances il souhaite faire assimiler à ses élèves et pourquoi, à quels apprentissages cela fait appel; il peut s'interroger sur la manière de progresser de la «matière première» de notre discipline, les lieux et les espaces identifiés, à son objet, l'espace organisé. L'enseignant sort alors du cadre strictement pédagogique et aborde à la fois ceux de l'épistémologie et de la didactique.

On ne saurait trop insister sur le fait qu'il est indispensable de fonder un enseignement sur une réflexion constante portant sur les tenants et aboutissants de la discipline en question. «Qu'est-ce que la géographie, comment a-t-elle évolué, pourquoi, sous quelles influences transdisciplinaires (...), que veut-elle montrer? A quelle intelligence du monde permet-elle d'accéder, par quelles démarches, par l'utilisation de quels outils?» (CLARY et RETAILLE 1986). Ce n'est qu'en se posant constamment ces questions qu'un maître peut être en mesure de concevoir un enseignement véritablement cohérent, quel que soit le programme dans le cadre duquel il travaille. La réflexion personnelle ne peut être remplacée par toutes sortes de recommandations didactiques ou méthodologiques élaborées par des tiers, aussi valables soient-elles: celles-ci risquent au contraire d'être un oreiller de paresse engendrant un enseignement stéréotypé et terriblement ennuyeux pour les élèves. La réflexion épistémologique ne peut donc être évacuée: c'est le rôle des institutions de formation quelles qu'elles soient (Universités ou institutions de formation pédagogique) de susciter, développer et entretenir cette réflexion sans laquelle l'enseignement risque bien de se résumer à des «recettes» plus ou moins bien appliquées et débouchant sur des résultats incertains.

Si l'enseignant a une idée assez précise de ce qu'est pour lui la géographie, il sait sans doute pourquoi il l'enseigne et connaît les contenus de son enseignement. Il pourra dès lors réfléchir au «comment» de cet enseignement. Quels problèmes va-t-il retenir, quels choix effectuera-t-il dans les programmes, quels fils conducteurs suivra-t-il, quels discours scientifiquement pertinents pourra-t-il tenir, quels objectifs se fixera-t-il? La démarche didactique est non seulement parallèle à la réflexion épistémologique, elle en est le complément obligé pour construire un enseignement cohérent.

Faut-il alors que tous les enseignants soient des chercheurs et des didacticiens? Non, mais on ne peut que souhaiter avec CLARY et RETAILLE que les maîtres soient «au moins en position de recherche avec leurs étudiants ou élèves. Que maîtres et élèves aient, ensemble, un problème à résoudre pour définir la méthode et les outils de la discipline, suivre une progression de démonstration, aboutir à une solution-explication. Cela constitue un modèle, une grille de référence et de lecture d'autres problèmes: c'est un apprentissage. La fonction de la didactique de la géographie est de montrer (...) les apprentissages qu'autorise la pratique de la discipline, de mettre en valeur la logique de la progression dans le savoir par la progression dans les apprentissages» (CLARY et RETAILlE 1986). Précisons encore que le savoir scolaire ne peut être un savoir universitaire dilué. Le savoir savant doit être transposé pour devenir objet d'enseignement et d'apprentissage. Le contenu d'un enseignement n'est pas un contenu scientifique appliqué, mais un contenu transposé. Là encore, la formation et le perfectionnement des maîtres, du point de vue didactique et méthodologique cette fois, ont un rôle essentiel à jouer pour que 
les enseignants soient prêts à entrer dans ce type de démarche.

On se gardera bien, enfin, d'évacuer l'élève de cette problématique! «Tout savoir se construit et ne s'inculque pas» (PLAGET et WALLON, cités par CLARY et RETAILLE 1986). L'élève construit son savoir à partir des contenus enseignés en classe, mais aussi à partir du savoir qu'il a prélablement acquis, à partir de sa sensibilité propre, de ses perceptions, de ses représentations. Ainsi en va-t-il pour l'espace qu'il vit, qu'il ressent, qu'il aime ou rejette. Et les contradictions pouvant surgir lorsque l'on confronte des approches individuelles fort différentes les unes des autres contribuent également à la construction de la connaissance, elles en sont un des moteurs. On doit donc prendre en compte non seulement les processus d'acquisition des connaissances, mais aussi la subjectivité, l'affectif de l'élève. L'affectif ne devrait pas être interpellé en guise de motivation initiale seulement, mais être présent tout au long de l'apprentissage, exception faite peut-être des nécessaires phases de conceptualisation.

Il faudrait dépasser largement le cadre de cet article pour examiner tous les programmes de géographie romands à la lumière des réflexions qui précèdent et cela demanderait l'intervention de leurs rédacteurs (membres de commissions cantonales de branches par exemple) pour qu'ils puissent préciser leurs intentions. Il ne s'agit donc pas ici d'allumer des querelles qui ne pourraient que nuire à la discipline. mais de constater que tous nos programmes sont perfectibles. Puissent simplement ces quelques idées servir de jalons pour la réflexion que devrait mener chaque enseignant... Et c'est encore CLARY et RETAILLE qui me permettront de conclure ces quelques observations et d'introduire un exemple de démarche qui en tient compte: "La conjonction de ces deux types de volonté, réflexion épistémologique et réflexion sur l'appropriation des connaissances, conditionne la transposition didactique et peut nous faire progresser vers une meilleure adaptation de l'enseignement de la géographie à la réalité du monde, de la classe, de l'élève. Nous affirmons que la géographie est tout autre chose qu'un vernis culturel, c'est un savoir qui a une utilité, une utilité sociale. Former à la géographie comme former aux mathématiques, c'est donner des clés de questionnement et de raisonnement, c'est rendre accessible la réponse à un problème: où, quoi, comment, pourquoi là?» (CLARY et RETAILLE 1986).

\section{Quelques pistes pour l'avenir...}

Travaillant dans la perspective d'une restructuration complète des programmes de géographie en Belgique, Mme MERENNE-SCHOUMAKER et ses collaborateurs proposent une démarche qui répond aux exigences formulées plus haut et semble apte à renouveler et à dynamiser l'enseignement de la discipline. En voici les grands principes. On veillera d'abord à organiser la connaissance progressive des différents ensembles spatiaux en s'appuyant sur les étapes de la conquête de l'espace par l'enfant, définies en 1969 déjà sur la base des travaux de la psychologie génétique (DEBESSE-ARVISET 1969). Rappelons ces étapes: de 8 à 9 ans environ, l'enfant découvre le milieu qui l'entoure, de 9 à 10 ans la région, de 9 à 11 ans le pays, de 10 à 12 ans le continent, de 12 à 13 ans les autres continents et de 12 à 15 ans environ le globe. Il s'agit donc du système désormais bien connu de la découverte de l'espace par cercles concentriques. Remarquons que les programmes romands ont adopté ce principe, ce qui est positif. MERENNE-SCHOUMAKER y ajoute cependant une étape: le milieu local ou régional doit être à la fois le point de départ et le point d'arrivée de la formation. Deuxième principe, à chaque niveau de la formation, les savoirs à acquérir doivent être définis en fonction des concepts de base de la discipline (localisation, distance, échelle, interaction, changement-permanence, espace géographique).

Troisième principe enfin: à chaque niveau de formation, les savoir-faire, les outils que l'élève doit mầtriser, doivent être précisés, ce qui implique une définition claire des objectifs généraux et des objectifs spécifiques de chaque étape de l'apprentissage. Dans ses travaux, MERENNE-SCHOUMAKER définit clairement ce qu'elle entend par savoir-faire géographique et objectifs de la géographie. Je renvoie le lecteur à son ouvrage cité en référence (MERENNESCHOUMAKER 1986).

Le fil conducteur d'un tel programme devrait être une boucle partant du milieu de vie des élèves pour y revenir à la fin de l'apprentissage. Ce qui est acquis en étudiant le milieu proche est utilisé comme outil pour la découverte du monde entier, cette découverte amenant elle-même de nouvelles acquisitions. L'élève est alors prêt à conceptualiser, à modéliser ses connaissances (son âge s'y prête), ce qu'il pourra faire au travers d'une séquence de «géographie générale». Enfin, il retourne à son milieu de vie; cette dernière étape n'est pas inutile: lorsque l'on revoit son milieu de vie après avoir vu le monde, on porte un autre regard sur son chez-soi...

Quant à la méthodologie proposée, applicable selon MERENNE-SCHOUMAKER à l'étude de n'importe quel espace, elle peut se résumer en trois grandes phases. La première est une phase de motivation: à partir de recherches sur l'espace vécu et perçu, on définit les questions à résoudre, par exemple par le biais d'un organigramme. La deuxième phase développe les connaissances et les savoir-faire par l'analyse de documents existants, ou la réalisation d'une enquête. La démarche et l'échelle d'analyse sont définies, les données traitées, les premières hypothèses expli- 
catives formulées, puis testées. On construit ainsi l'explication. La troisième phase enfin permet le développement du savoir-faire de l'élève. En confrontant les résultats de la deuxième phase avec les perceptions mises en évidence lors de la première phase, on peut, après intégration des deux groupes de données ou rejet de cette intégration, élaborer des solutions au problème étudié et choisir une ou plusieurs solutions: on peut déboucher ainsi sur une action personnelle de l'élève, ou une action collective de la classe et l'on contribue alors au développement du savoir-être de l'élève. Le rôle du maître varie au cours de ces trois phases: d'animateur-coordinateur dans la première phase, il devient expert dans la seconde et éducateur dans la troisième.

Ce type de méthodologie vise donc à faire aboutir à un résultat concret les recherches effectuées en classe. Elle prépare l'élève à l'action et facilite en même temps l'apprentissage du dialogue et de la participation. C'est là incontestablement un moyen de prendre aussi souvent que possible l'affectif de l'élève en compte, et de former les adultes de demain au rôle de décideur qui sera le leur. Je reviendrai dans ma conclusion sur l'importance de cette géographie de l'action.

Une telle géographie pourrait être introduite par le biais des jeux de simulation ou des jeux de rôles. Pratiqués depuis longtemps par les géographes anglo-saxons (que l'on pense aux livres d'un Walford, par exemple, ou à la démarche de l'Oxford Geography Project), ils ne bénéficient pas encore, et de loin, d'une semblable audience au niveau scolaire dans l'aire francophone, même si des groupes de maîtres développent leur emploi ici et là. Mais ils restent des cas relativement isolés et se heurtent souvent à des réactions de méfiance des parents ou des autorités scolaires, pour qui le mot «jeu» implique qu'il ne peut s'agir d'une activité «sérieuse»... Et pourtant, les jeux de simulation et les jeux de rôles permettent un apprentissage direct, vécu, de la notion de processus; ils favorisent l'acquisition et l'utilisation du vocabulaire, la compréhension des mécanismes complexes et des lois. Ils sont un moyen de sortir de l'approche descriptive, statique, en mettant en valeur le fonctionnement des interactions dans le temps.

Enfin, une nouvelle tendance de la géographie semble prometteuse, bien qu'elle ne soit pour l'instant pratiquée que par de petits groupes d'enseignants. Il s'agit de la géographie des représentations, dont un ouvrage récent propose des exemples d'utilisation dans le cadre scolaire (CLARY et al. 1987). Cette démarche, qui consiste à analyser les représentations que l'on se fait de l'espace, pourrait être le point de départ d'un travail aboutissant ensuite aux deuxième et troisième étapes de la méthode proposée par MERENNE-SCHOUMAKER et débouchant ainsi sur une action concrète. Encore une fois, la géogra- phie ne peut se justifier qu'en tant que discipline qui a une utilité, une utilité sociale: ce n'est pas en en faisant un vernis culturel que l'on conférera cette utilité à la géographie, mais en lui permettant de mener à l'action concrète et réfléchie.

\section{En guise de conclusion}

Les réflexions qui précèdent et les idées - très générales - qu'elles proposent sont-elles compatibles avec la pratique, avec la réalité scolaire d'aujourd'hui, ou ne sont-elles que des vœux pieux plus ou moins utopiques? Il est certain que la mise en pratique réelle de telles idées passe par des gens convaincus et formés à ces démarches. Or, la géographie est encore très et trop souvent enseignée par des maîtres qui n'ont pas de formation spécifique dans cette discipline: dans le canton de Vaud par exemple, seuls $11 \%$ des maîtres de géographie travaillant en 1988 dans l'enseignement secondaire inférieur sont des spécialistes formés à l'Université. Même si ce chiffre doit être relativisé en raison de la nouvelle structure de l'enseignement secondaire vaudois, il ne manque pas de laisser songeur... Comment peut-on raisonnablement exiger d'un nonspécialiste qu'il mène une réflexion épistémologique poussée, alors qu'il a plusieurs autres branches à enseigner... et que certains spécialistes se contentent du confort de la routine sans jamais s'interroger? La possibilité existe-t-elle pour une seule personne d'assurer un enseignement de qualité dans plusieurs domaines? La question est grave et mérite d'être soulevée, car elle remet en évidence de nombreux problèmes liés à la formation et au perfectionnement des maîtres. Ces domaines ont-ils vraiment le soutien politique et financier nécessaire? N'y a-til pas là des questions fondamentales, à résoudre d'urgence (on sort d'ailleurs ici du cadre strict de la géographie!)? Les réponses ne sont pas cette fois-ci de la seule compétence des géographes. Ceux-ci doivent cependant être capables de démontrer l'utilité de leur discipline aux décideurs d'aujourd'hui, et cette tâche n'admet plus de retard.

Lorsque l'on connaît les enjeux auquel est soumis l'espace, qui voit s'affronter les intérêts contradictoires des milieux agricoles, industriels, immobiliers, touristiques, des producteurs d'énergie, des entreprises de transport, des défenseurs de l'environnement (la liste n'est pas exhaustive!), lorsque l'on sait que la compétition est toujours plus vive entre ces différents «agents spatiaux» et qu'elle le sera encore plus dans l'avenir, on mesure l'attention primordiale que l'on doit accorder à la formation, à l'«éducation géographique» des jeunes, qui demain devront gérer l'espace que nous aurons produit et qui seront appelés à leur tour à opérer des choix fondamentaux à partir de cet espace et pour lui. 
Dans l'enseignement en général et secondaire en particulier, la géographie peut et doit être une discipline qui mette en place non seulement un savoir et un savoir-faire, mais encore un savoir-être, le «savoir penser l'espace», pour reprendre la célèbre expression de LACOSTE (LACOSTE 1984). Savoir penser l'espace, c'est apprendre à mâttriser le milieu de vie en établissant avec lui des rapports équilibrés, en fonction de ses mécanismes et des conditions de développement optimum de l'Homme, pour être en mesure d'y agir efficacement. Ce «savoir penser l'espace» serait d'ailleurs le lieu idéal d'un travail pluridisciplinaire, la géographie ne se contentant plus alors, comme elle le faisait trop souvent, d'emprunter à d'autres disciplines ce qu'elles font mieux qu'elle, mais apportant sa spécificité même au travail commun.

C'est sans doute cette dernière dimension, celle du «savoir penser l'espace» permettant de déboucher sur une maîtrise du milieu de vie, qui est en pratique la plus absente des programmes scolaires et des contenus d'enseignement, même si elle figure souvent au rang des objectifs généraux de la discipline. Il y a certainement nombre d'explications à cet état de faits, qui vont du poids de la tradition scolaire aux pressions politiques. Il est temps de briser ces résistances pour que l'utilité de la géographie scolaire - et de la géographie en général! - soit enfin reconnue à sa juste valeur, pour que la discipline trouve ainsi une place solide dans les grilles de programmes, et bénéficie dans le même temps des moyens matériels et humains (formation et perfectionnement surtout) qui lui sont nécessaires. Audélà de l'amélioration du statut scolaire de la discipline, il n'est pas exagéré de penser que ce combat est aussi celui qu'il faut mener pour éveiller la conscience de tout un chacun, et particulièrement des jeunes, aux mesures à prendre pour que notre milieu de vie demeure... vivable.

\section{Zusammenfassung}

Dieser Artikel stellt die grundlegenden Tendenzen der heutigen Schulgeographie in der Westschweiz dar. Ein kurzer Überblick über die Lehrpläne der Geographie in der Romandie dient als Grundlage für einige didaktische Überlegungen. Dabei wird offensichtlich, daß die aktuelle Schulgeographie eine Vorbereitung für Handlungen sein sollte, die zu einer Beherrschung des Lebensraumes führen. Diese Forderung wird durch einige konkrete Vorschläge unterstützt.

\section{Références}

CLARY, M., DUFAU, G., DURAND, R., FERRAS, R. (1987): Cartes et modèles à l'école, Montpellier.

CLARY, M., RETAILLE, D. (1986): Une nouvelle rubrique de I'Information Géographique, Didactique de la géographie. In: L'Information Géographique, no 50, 36.

DAUDEL, C. (1986): La recherche en didactique de la géographie: réflexions méthodologiques pour une investigation scientifique. In: Revue de Géographie de Lyon, 1986/2, 133-157.

DEBESSE-ARVISET, M.-L. (1969): La géographie à l'école, Paris.

LACOSTE, Y. (1984): La géographie et l'histoire. In: L'Information Géographique, no 48, 71-74.

MERENNE-SCHOUMAKER, B. (1986): Eléments de didactique de la géographie. In: G.E.O., no 19 (numéro spécial), $1-135$.

MERENNE-SCHOUMAKER, B. (1988): Un schéma directeur pour l'enseignement de la géographie aujourd'hui: fondements, principes et moyens, applications. Article inédit et texte d'une conférence donnée dans le cadre du cours CPS "Aspects de la didactique de la géographie", à Lausanne, 30 mai-1er juin 1988.

Programmes officiels de géographie des cantons romands, publiés par les différents Départements de I'Instruction Publique. Ces documents m'ont été remis par les Départements en question ou par des maîtres de didactique et de méthodologie de la géographie travaillant dans les cantons concernés.

En outre, les trois revues suivantes ont consacré une large place à la didactique de la géographie dans certains de leurs numéros, en particulier:

L'Espace Géographique, no 1/1986, 4-47, et no 2/1986, $131-154$.

L'Information Géographique, 1986, no 50, 36, 74-88, 150-151, 167-168.

Revue de Géographie de Lyon, no 1986/2, 132-242.

A signaler enfin l'excellente revue publiée par la FEGEPRO, la Fédération des Professeurs de Géographie de Belgique, revue intitulée G.E.O.

Remerciement: je tiens à exprimer ici toute ma gratitude à mon épouse, et plus particulièrement pour son aide précieuse lors de la dactylographie du manuscrit. 